\section{Case Report: Spontaneous Coronary Artery Dissection in a Post Partum 30 Year Old Female}

\section{Abstract}

Spontaneous coronary artery dissection in post-partum patients is a rare and potentially life-threatening event observed in otherwise healthy, young women. This case discusses a multi - vessel coronary artery dissection in a female 12 days post-partum. This article discusses the phenomenon of spontaneous coronary artery dissection in postpartum patients, emphasizing the important of raising awareness on these unique cases.

Keywords: Coronary artery; Myocardial Infarction; Cardiology; Dissection; Postpartum

Received: November 23, 2015, Accepted: December 22, 2015, Published: December 29, 2015

\section{Hany Y Atallah ${ }^{1}$, Ashleigh M Magill², Brooks Moore ${ }^{3}$, Jeremy Nelson ${ }^{4}$ and Michael McDaniel ${ }^{5}$}

\author{
Chief of Emergency Medicine, Emory \\ University, USA
}

Corresponding author: Hany Y Atallah

\section{hatalla@emory.edu}

MD, FACEP, Chief of Emergency Medicine, Grady Health System, Associate Professor, Department of Emergency Medicine, Emory University, USA

Tel: $678-707-4164$

\section{Case Report}

A 30 - year - old African American female, P4004, presented to the emergency room 12 days postpartum with the chief complaint of chest pain. The pain had been gradually worsening over three days, and was described as a substernal pressure with intermittent radiation into the neck. The patient had a history of hypertension for which she was prescribed triamterene - hydrochlorothiazide, but did not require antihypertensive therapy during pregnancy and had not resumed her medication postpartum. Review of systems otherwise revealed an occasional headache unrelated to chest pain.

Her initial EKG (Figure 1) did not reveal any ST segment abnormalities. Her blood pressure on arrival was $175 / 100$, and she was treated initially with $15 \mathrm{mg}$ IV Hydralazine. Her labs revealed an elevated troponin - I level of 0.83 . Urinalysis was negative for protein, making pre - eclampsia unlikely. She continued to have constant chest pain and hypertension. A repeat EKG at this time (Figure 2) revealed ST segment depression in leads II and III with a tall $\mathrm{R}$ wave in V2 suggesting possible posterior wall myocardial infarction. Nitroglycerin paste, aspirin and morphine were given. Repeat troponin at this time was sent and found to be 4.16. A CT angiogram of the neck, chest, and abdomen/pelvis, performed to rule out aortic dissection, was negative. The patient was admitted for further management of hypertensive crisis and Non - ST Elevation Myocardial Infarction (NSTEMI).

Overnight, her troponin peaked at 16; a transthoracic echocardiogram (TTE) revealed a normal ejection fraction (EF) with posterior wall hypokinesis. The patient was started on heparin and taken for cardiac catheterization the following morning. Diagnostic catheterization revealed significant multivessel coronary artery dissection involving the mid-left circumflex (LCX) artery ( $90 \%$ stenosis), mid-distal left anterior descending (LAD) artery ( $80 \%$ stenosis) and right coronary artery (RCA) (80\% stenosis) (Figures 3-5). The patient was immediately taken to the operating room for an emergent on-pump triplevessel coronary artery bypass graft (CABG).

Post - operatively the patient experienced recurrence of chest pain, and ruled in for repeat MI. A repeat coronary catheterization demonstrated early graft vein failure of the RCA graft. She was medically managed antiplatelet and anticoagulation treatment. Her symptoms improved and her troponin - I trended downward. Catheterization prior to discharge showed patency of the LCX and LAD grafts, complete stenosis of the RCA graft, and overall improvements of native vessel disease with TIMI - 3 flow. 

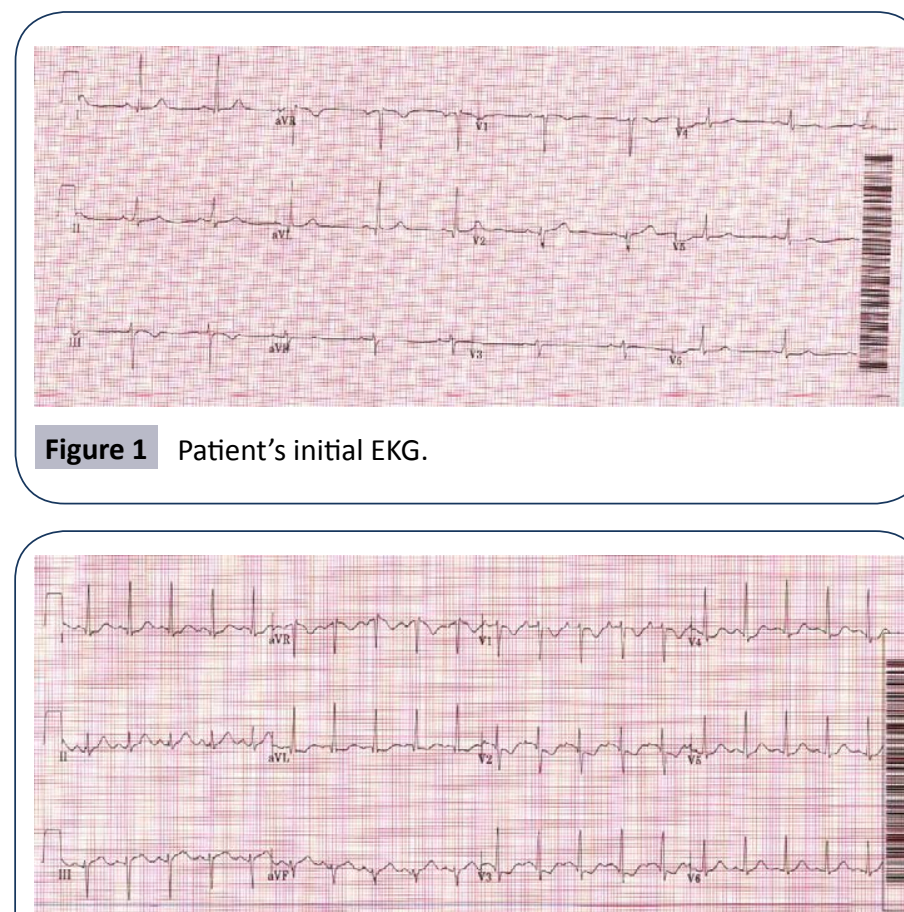

Figure 2 Repeat EKG done approximately $4 \frac{1}{2}$ hours after the first EKG.

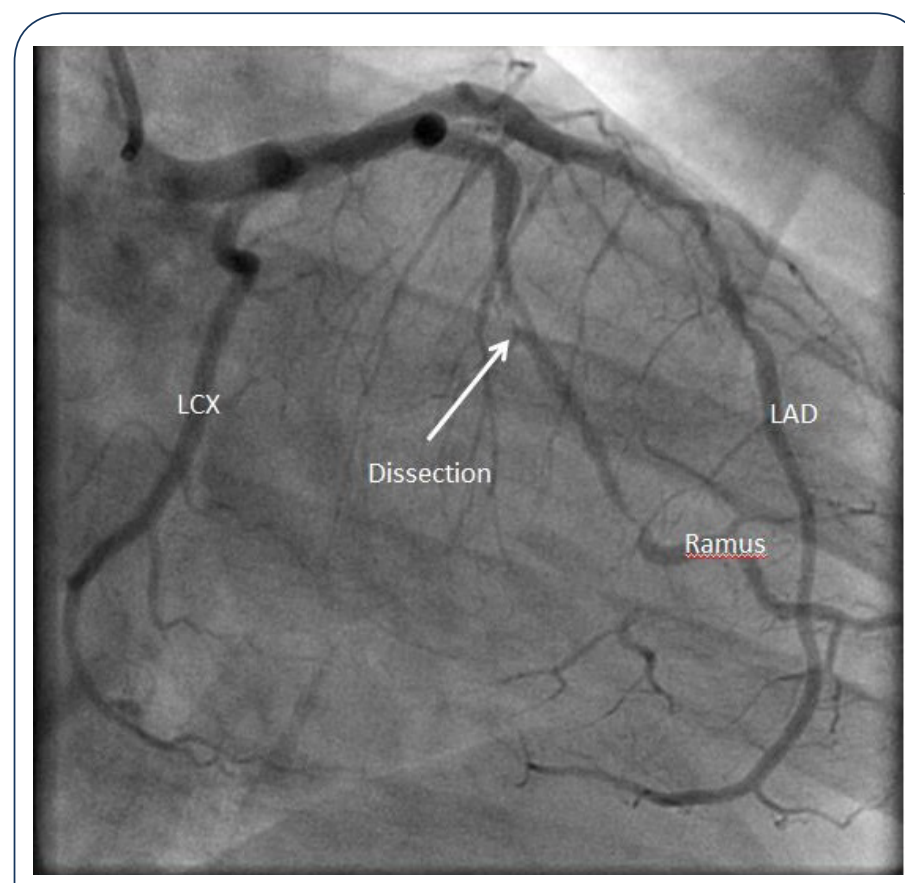

Figure 3 Cardiac catheterization image showing a dissection of the ramus coronary artery.

Echocardiogram prior to discharge demonstrated an EF of 40$45 \%$ and ongoing, but improved, left ventricular (LV) hypokinesis.

\section{Discussion}

Spontaneous coronary artery dissection (SCAD) accounts for a small, yet significant, subset of patients with pregnancyassociated coronary artery disease. Occurring in young, generally healthy women during pregnancy and the postpartum period, it

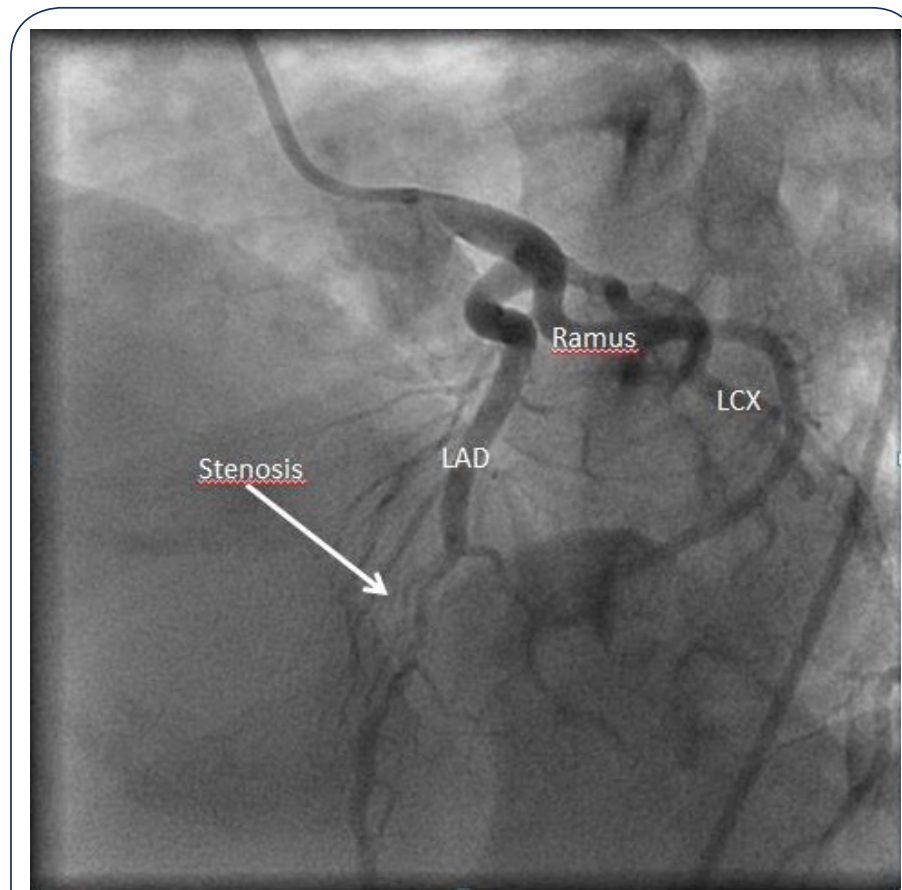

Figure 4 Cardiac catheterization image showing a dissection of the left anterior descending coronary artery (LAD).

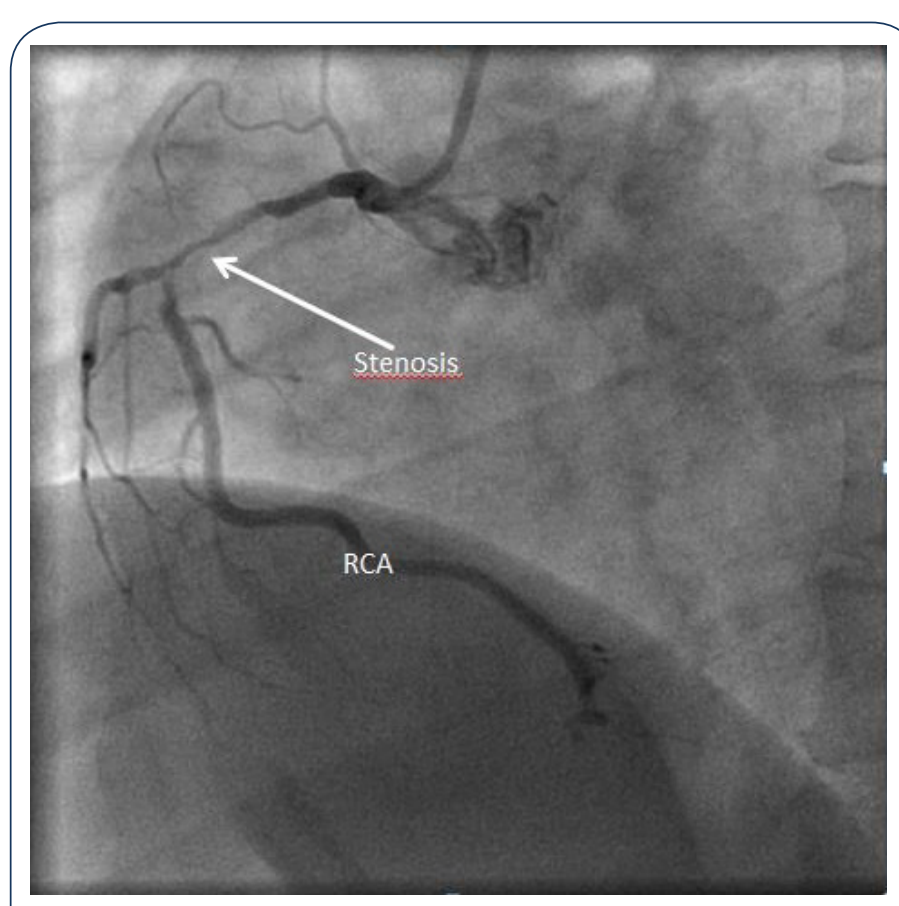

Figure 5 Cardiac catheterization image showing a dissection of the right coronary artery (RCA).

is associated with up to $38 \%$ mortality if not recognized, but an almost $100 \%$ survival rate after early identification [1,2]. This is especially important given that these patients can present with an array of symptoms ranging from substernal chest pain to cardiogenic shock [3]. The case discussed here is a prime example of an initially well-appearing patient who was subsequently found to have severe disease, which may have not been detected had blood-work not been attained.. 
As of 2008 there were 73 case reports in the literature, reviewed by Koul et al. and Appleby et al. pertaining to coronary artery dissection in pregnancy and the postpartum period [1,2]. Review of these cases demonstrated an increased frequency in older (age greater than 30) and multiparous patients, with pre-existing risk factors for $C A D$ observed in around one-third of these patients. These reviews also concluded the majority of SCADs are singlevessel, and predominantly (80\%) involve the LAD [1].

The etiology of these events is suspected to be multifactorial. Some postulate increased cardiac output secondary to the gravid state is a contributor, which may predispose patients to dissection [4]. A popular theory, which has been mentioned in multiple reports, is that of excess circulating estrogen triggers eosinophils to release proteases, resulting in compromised vessel integrity $[5,6]$. There have been isolated case reports of postpartum dissection in women who were found to have high titers of anticardiolipin antibody; however, this has not been regularly tested in the majority of women presenting with SCAD $[7,8]$. Given the myriad physiologic changes that occur in the gravid and postpartum state it is more important to recognize the impact of these processes with regards to increased risk for dissection, rather than to identify an isolated etiology.

During evaluation of pregnant and post-partum patients in the emergency room, it is important to remember AMI and SCAD as parts of the differential. With regards to imaging, catheterization remains the gold standard for identifying dissection, although some case reports using transesophageal echocardiogram (TEE) have been described [5]. Since the initial case report by Pretty in 1931, there have been vast improvements in both the diagnosis and management of all acute coronary syndromes, with more recent literature reviews showing a trend for earlier catheterization in postpartum AMI patients -- perhaps also explaining the decline in mortality rates. Early catheterization has also been crucial in dictating management in patients, as only $10 \%$ of all post-partum patients with AMI have SCAD [5].
Management of SCAD in postpartum patients presents a challenge to the physician. In patients with single-vessel disease, medical management is first line, consisting of aspirin, nitrates, beta-blockers and heparin $[2,9]$. While this was successful in some patients, it was less likely to be sufficient in those with multivessel disease or those who experienced ongoing symptoms related to single-vessel disease $[10,11]$. Patients with multi-vessel involvement or those who fail medical therapy often require more invasive management options, with guidelines for CABG being similar to those of patients with CAD [5]. Thrombolytics have been used in select case reports, but given the nature of this process is not thrombotic, and there have been negative outcomes associated with their use, it is at the discretion of the physician managing the patient whether or not to initiate these in the emergency department [12].

\section{Why should an emergency physician be aware of this?}

With the advent of coronary artery stenting, the management of these cases has improved, resulting in improved mortality for these patients. However, one crucial component in these cases has been consistent and that is the early recognition and diagnosis of SCAD. As previous reports suggest, the number of cases reported may not accurately reflect the true prevalence when definitive diagnosis relies on catheterization. It is critical for physicians to consider AMI and SCAD in their differential when evaluating postpartum patients, and to encourage early catheterization when presented with possible SCAD.

\section{Conclusion}

At one-month follow up the patient had experienced no recurrence of symptoms, and tolerating was medical management well. At 4 month follow up a repeat echocardiogram demonstrated an EF of $35-40 \%$, mild dilation of LV with a noted restrictive fill-pattern. 


\section{References}

1 Appleby CE, Barolet A, Ing D, Ross J, Schwartz L, et al. (2009) Contemporary management of pregnancy-related coronary artery dissection: A single - centre experience and literature review. Exp Clin Cardiol 14: e8-e16.

2 Koul AK, Hollander G, Moskovits N, Frankel R, Herrera L, et al. (2001) Coronary artery dissection during pregnancy and the postpartum period: two case reports and review of literature. Catheter Cardiovasc Interv 52: 88-94.

3 Goland S, Schwarz ER, Siegel RJ, Czer LS (2007) Pregnancy-associated spontaneous coronary artery dissection. Am J Obstet Gynecol 197: e11-3.

4 Roth A, Elkayam U (2008) Acute myocardial infarction associated with pregnancy. J Am Coll Cardiol 52: 171-80.

5 Frey BW, Grant RJ (2006) Pregnancy - associated coronary artery dissection: a case report. J Emerg Med 30: 307-310.

6 Borczuk AC, van Hoeven KH, Factor SM (1997) Review and hypothesis: the eosinophil and peripartum heart disease (myocarditis and coronary artery dissection) - coincidence or pathogenetic significance? Cardiovasc Res 33: 527-532.
7 Kiernan TJ, Rochford M (2007) Postpartum spontaneous coronary artery dissection: an important clinical link with anticardiolipin antibody. Int J Cardiol 114: E75-76.

8 Krishnamurthy M, Desai R, Patel H (2004) Spontaneous coronary artery dissection in the postpartum period: association with antiphospholipid antibody. Heart 90: e53.

9 Kalra N, Greenblatt J, Ahmed S (2008) Postpartum spontaneous coronary artery dissection (SCAD) managed conservatively. Int J Cardiol 129: e53-5.

10 Azzarelli S, Fiscella D, Amico F, Giacoppo M, Argentino V, et al. (2009) Multivessel spontaneous coronary artery dissection in a postpartum woman treated with multiple drug-eluting stents. J Cardiovasc Med (Hagerstown) 10: 340-343.

11 Bicer M, Saba B, Ozdemir B, Ercan A (2008) Idiopathic spontaneous coronary artery dissection: a case report. Thorac Cardiovasc Surg 56: 486-488.

12 Eby CS, McBride LR (2001) Postpartum coronary artery dissection complicated by heparin-induced thrombocytopenia and thrombosis. Ann Thorac Surg 71: 1025-1027. 